\title{
Statt eines Schlussworts eine Bitte
}

Die Erstellung dieses Buches hat acht Monate gedauert. Nicht mitgerechnet sind all die Jahre, in denen ich Anwendungsbeispiele gesammelt und teilweise in meinen anderen Büchern beschrieben habe. Was mir dabei immer wieder auffiel, ist, dass ScoringModelle bzw. NWA nicht etwa nur betriebswirtschaftliche Entscheidungsverfahren sind, sondern Denkmuster repräsentieren. Ein komplexes Problem in kleine Teile zu zerlegen ist ein „Denktrick“, den bereits die griechischen Klassiker beschrieben und der mir in den didaktischen Schriften René Descartes wiederbegegnet ist. Dieses Muster für betriebswirtschaftliche Zwecke zu beschreiben und mit den erforderlichen Verfahrensregeln zur Ausgestaltung der Priorisierung und Bewertung von Kriterien zu verfeinern, ist der Verdienst Zangemeisters. Jedenfalls hat er es in den 70er Jahren in Deutschland populär gemacht und in den letzten knapp 50 Jahren hat sich das Scoring bzw. die NWA etabliert.

Mein Beitrag bestand darin, den Stand der methodischen Entwicklung zu beschreiben und zahlreiche Anwendungsfälle mit all ihren Besonderheiten zu diskutieren. Vor allem habe ich versucht, die bei der konkreten Anwendung zwangsläufig aufkommenden methodischen Detailfragen zu beantworten: Welche Skala, welche Gewichtung, welche Bewertung ... all das liest sich im Managementhandbuch einfach, aber bei der Umsetzung entstehen viele Fragezeichen, denn der Teufel steckt wie immer im Detail.

Vermutlich habe ich einige Einsatzbereiche übersehen, aber ich hoffe, dass ich hinsichtlich der Besonderheiten alles Wichtige berücksichtigt habe. Wenn nicht, und das ist meine Bitte, schicken Sie mir einen Hinweis, damit ich es für die nächste Auflage besser machen kann! 